\title{
Model analysis of temperature dependence of abnormal resistivity of a multiwalled carbon nanotube interconnection
}

This article was published in the following Dove Press journal:

Nanotechnology, Science and Applications

20 July 2010

Number of times this article has been viewed

\section{Yi-Chen Yeh' \\ Lun-Wei Chang² \\ Hsin-Yuan Miao ${ }^{3}$ \\ Szu-Po Chen' \\ Jhu-Tzang Lue'}

'Department of Physics and ${ }^{2}$ Institute of Electronics Engineering, National Tsing Hua University, Hsinchu, Taiwan;

${ }^{3}$ Department of Electrical Engineering,

Tunghai University, Taichung, Taiwan
Correspondence: HY Miao Department of Electrical Engineering, Tunghai University, I8I Taichung Harbor Road, Section 3, Taichung, 40704, Taiwan $\mathrm{Tel}+886423590 \mathrm{O} 2 \mathrm{I}$ extn 33902

$\mathrm{Fax}+88635743052$

Email kenymiao@thu.edu.tw
Abstract: A homemade microwave plasma-enhanced chemical vapor deposition method was used to grow a multiwalled carbon nanotube between two nickel catalyst electrodes. To investigate the transport properties and electron scattering mechanism of this interconnection (of approximately fixed length and fixed diameter), we carried out a model analysis of temperature dependence of resistivity. To explain the abnormal behavior of the negative temperature coefficient of resistivity in our experimental results, we then employed theories, such as hopping conductivity theory and variable range hopping conductivity theory, to describe resistivity in the high- and low-temperature ranges, respectively. Further, the grain boundary scattering model is also provided to fit the entire measured curve of temperature dependence of resistivity.

Keywords: multiwalled carbon nanotube, resistivity, hopping conductivity, temperature dependence

\section{Introduction}

Nanoscale electronic devices are expected to become more and more integral to future high-performance integrated circuit devices, due to their intrinsic properties regarding latency and power dissipation. To further advance silicon-integrated circuit (IC) technology, the high-speed transmission needs of chips must be taken into account, and the novel properties of carbon nanotubes (CNTs) show exciting promise for leading future IC chip interconnect developments. ${ }^{1}$ The extremely low density of CNTs as solids, ie, $1.3-1.4 \mathrm{~g} / \mathrm{cm}$ means their specific strength is up to $48,000 \mathrm{kN} / \mathrm{m} / \mathrm{kg}$, which is the best specific strength of all known materials, comparing well with the specific strength of high-carbon steel, ie, $154 \mathrm{kN} / \mathrm{m} / \mathrm{kg}$. ${ }^{2}$ In theory, metallic nanotubes can carry an electrical current density of $4 \times 10^{9} \mathrm{~A} / \mathrm{cm}^{2}$, which is more than 1000 times greater than that able to be carried by copper. While their electronic transport properties have attracted significant interest, ${ }^{3-5}$ CNTs might also play a pivotal role in the fabrication of electronic nanoscale devices due to their unique mechanical flexibility, ${ }^{6}$ resistance to chemical corrosion, ${ }^{7}$ and high field emission properties. ${ }^{8}$

These exciting properties are dependent on the structure and synthesis of the CNTs. Theoretically, in pure metallic single-walled carbon nanotubes (SWCNTs) or multiwalled carbon nanotubes (MWCNTs), electron transport would occur ballistically, such that electrons would transport along the nanotube axis without any power dissipation due to scattering. ${ }^{9,10}$ Thus, electrons and phonons would propagate easily along the same tube axis.

However, in practice, there are many structural factors involving the synthesis of CNTs to consider, eg, defects (impurity, vacancy) or grain boundary in/on the CNTs. 
Extensive research and literature are devoted to understanding and finding solutions for improving CNT structural defects and problems. Naeemi and Meindl used equivalent circuit models to simulate various electron-phonon scattering mechanisms as a function of temperature and to model the temperature coefficient of resistance (TCR) for different types, defects, and lengths of CNTs. ${ }^{11}$ Resistivity is influenced by certain defects (such as Stone-Wales defects and the Aharonove-Bohm effect), as well as catalysts remaining in the CNTs that cause extra scattering and interface roughness. ${ }^{12,13}$ Structure defects of the material, such as interface roughness and small grain size, also cause variations of the electron transport characteristics. ${ }^{8}$ Self-heating also plays a significant role in short-length nanotubes transporting current under high bias. ${ }^{14}$ Svizhenko et al reported that "large diameter nanotubes are preferable because even the semi-conducting shells can carry current due to small band gaps", and "the conductance decrease with increase in bias was caused by reflection of incident electrons at crossing subbands due to scattering with zone boundary phonons". ${ }^{15}$

In our work, using the concept of phenomenology, we attempt to fit a temperature dependence of resistivity model to the experimental high-to-low temperature range (300-25 K), with results from an individual MWCNT (of approximately fixed length and diameter) shown in Figure 1. This particular MWCNT was fabricated using a homemade microwave plasma-enhanced chemical vapor deposition (MPECVD) system. ${ }^{4,16}$ In order to explain the result of a negative TCR, we took into account the theories of hopping conductivity, variable range hopping conductivity, strong localization $\left(\mathrm{k}_{\mathrm{f}} \mathrm{l}_{0} \sim 1\right.$, in the product $\mathrm{k}_{\mathrm{f}} 1_{0}$, where $\mathrm{k}_{\mathrm{f}}$ is the quasi-Fermi wave vector, $1_{0}$ is a finite mean free path, and reflects the degree of the disorder and localization) and grain boundary scattering. ${ }^{17}$

\section{Theory}

\section{Hopping conductivity}

If the number of impurities or defects in the crystalline structure increases, then electron wave function spans only a few lattices and fades exponentially. ${ }^{18}$ Therefore, the conductivity is varied due to electron tunneling between localized states, and a negative TCR arises from phonon-assisted hopping conduction. Excitation of electrons to $E_{c}$ which contributes to conductivity by hopping can be expressed as strongly localized states:

$$
\boldsymbol{\sigma}=\boldsymbol{\sigma}_{\min } \exp \left(-\frac{\boldsymbol{E}_{\boldsymbol{c}}-\boldsymbol{E}_{\boldsymbol{F}}}{\boldsymbol{k} \boldsymbol{T}}\right)
$$
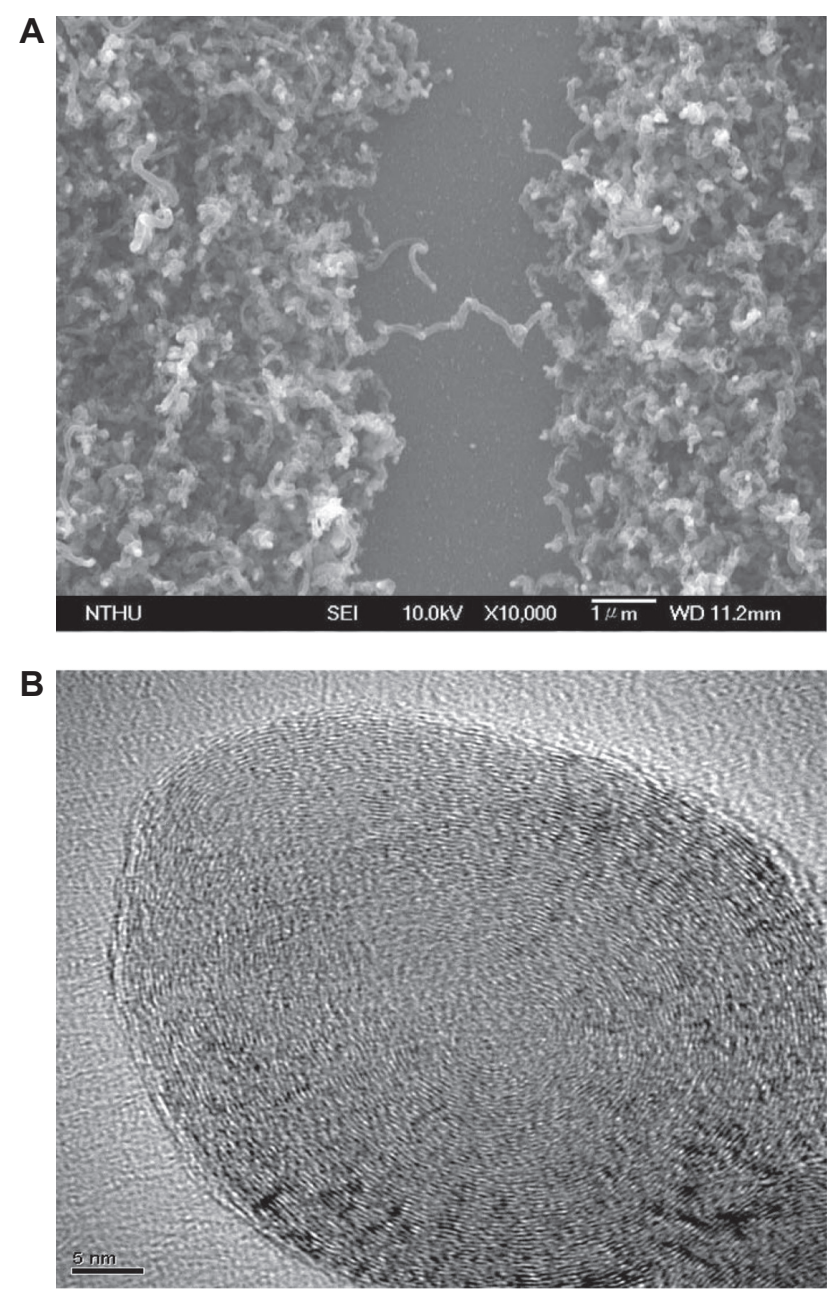

Figures I A) The scanning electron microscopic image of a multiwalled carbon nanotube which was grown at $650^{\circ} \mathrm{C}$ and $10^{-5}$ torr with a $3 \mu \mathrm{m}$ length and $100 \mathrm{~nm}$ diameter between two isolated electrodes as a current conduction bridge. . $^{416}$ B) A cross-sectional image of another sample of the same batch of multiwalled carbon nanotubes.

Where $\boldsymbol{E}_{\boldsymbol{c}}$ is the mobility edge and $\boldsymbol{E}_{\boldsymbol{F}}$ is the Fermi energy. This form of conduction is normally predominant at relatively high temperatures, or when $\left(\boldsymbol{E}_{c}-\boldsymbol{E}_{\boldsymbol{F}}\right)$ is small (for small value of $\boldsymbol{E}_{c}-\boldsymbol{E}_{F}$, the transport path and the typical hopping distance are defined, respectively). ${ }^{19}$

\section{Variable range hopping}

Thermally activated, variable range hopping conduction by electrons in the states near the Fermi energy at low temperatures $^{18}$ is described as:

$$
\boldsymbol{\sigma} \approx 2 \boldsymbol{e}^{2} \boldsymbol{R}^{2} \boldsymbol{v}_{p h} \boldsymbol{N}\left(\boldsymbol{E}_{F}\right) \exp \left(-\frac{\boldsymbol{B}}{\boldsymbol{T}^{1 / d+1}}\right)
$$

Where $\boldsymbol{R}$ is the hopping distance, $v_{p h}$ denotes the phonon energy, $\boldsymbol{B}$ is the coefficient related to the density of state, and $d$ indicates the dimension. This condition occurs at sufficiently low temperatures. 


\section{Grain boundary scattering}

Mayadas and Shatzkes were the first researchers who explored the theory of boundary scattering of electrons in thin, polycrystalline, metal films. ${ }^{20}$ However, Hoffmann et al empirically proposed a superior method to take into account and explain the boundary scattering of electrons. ${ }^{21}$ The conductivity of thin, polycrystalline films strongly deviates from the conductivity of its corresponding bulk single crystalline materials. Hoffmann et al stated that the reduction of conductivity depends exponentially on the number of grain boundaries per mean free path (MFP), and all electrons reflected by the grain boundaries along one mean free path do not contribute to the resulting current. The DC conductivity of polycrystalline films can be assumed as:

$$
\boldsymbol{\sigma}_{g}=\frac{\boldsymbol{n} \boldsymbol{e}^{2} \boldsymbol{l}_{\infty}}{\boldsymbol{m} \boldsymbol{v}_{F}} \boldsymbol{T}_{g}^{\frac{l_{\infty}}{R_{G}}}
$$

Where $\boldsymbol{l}_{\infty} / \boldsymbol{R}_{G}$ is the number of grain boundaries per mean free path and $\boldsymbol{T}_{\mathrm{g}}$ is the average probability for an electron to tunnel a single grain boundary, such that the effective mean free path can be expressed as:

$$
\boldsymbol{l}_{g}=\boldsymbol{l}_{\infty} \cdot \boldsymbol{T}_{g}^{\frac{\boldsymbol{l}_{\infty}}{R_{G}}}
$$

where $\boldsymbol{l}_{\infty}{ }^{-1}=\boldsymbol{l}_{o}^{-1}+\boldsymbol{l}_{i n}{ }^{-1}$ is due to Matthiessen's law. ${ }^{22}$ Here, $\boldsymbol{l}_{\boldsymbol{o}}$ is the elastic mean free path resulting from acoustic phonon scattering, while the temperature dependence of inelastic mean free path $\boldsymbol{l}_{\text {in }}$ is expressed as $\boldsymbol{l}_{i n}^{-1}(\boldsymbol{T}) \propto \boldsymbol{T}^{P}$, which is estimated approximately by Bloch-Grüneisen's law and then can be assumed as $\boldsymbol{l}_{o} / \boldsymbol{l}_{i n}(\boldsymbol{T})=\boldsymbol{b} \boldsymbol{T}^{P}$. If the grain size is $\boldsymbol{r}_{G}$, the crystalline proportion inside the film can be denoted as $\left(\mathbf{r}_{\mathrm{G}} / \boldsymbol{R}_{G}\right)^{3}$. Therefore, the conductivity can be revised as

$$
\boldsymbol{\sigma}_{g}=\frac{\boldsymbol{n} \boldsymbol{e}^{2} \boldsymbol{l}_{o}}{\boldsymbol{m} \boldsymbol{v}_{F}} \cdot \frac{1}{1+\boldsymbol{b} \boldsymbol{T}^{P}} \cdot\left(\frac{\boldsymbol{r}_{G}}{\boldsymbol{R}_{G}}\right)^{3} \boldsymbol{T}_{g}^{\frac{l_{o}}{R_{G}\left(1+b T^{P}\right)}} .
$$

Furthermore, we can finally obtain the resistivity:

$$
\boldsymbol{\rho}=\frac{\boldsymbol{m} \boldsymbol{v}_{F}}{\boldsymbol{n} \boldsymbol{e}^{2} l_{o}}\left(1+\boldsymbol{b} \boldsymbol{T}^{P}\right)\left(\frac{\boldsymbol{R}_{G}}{\boldsymbol{r}_{G}}\right)^{3} \boldsymbol{T}_{g}^{-\frac{l_{o}}{R_{G}\left(1+b T^{P}\right)}} .
$$

Thus, depending on the choice of transmission coefficient $T$ and the grain size $\boldsymbol{r}_{G}$, using the temperature dependence of grain boundary scattering theory can produce a positive or negative TCR.

\section{Experimental design}

In this study, for the purpose of modeling an analysis of the temperature dependence of resistivity, a MWCNT was fabricated as an interconnector by the MPECVD method. ${ }^{4,16}$ Catalyst nickel electrodes were deposited onto a $\mathrm{SiO}_{2}$ layer which was grown on a silicon wafer by the traditional thermal oxidation system to prevent current leakage from the MWCNT device. The gap between the electrodes was approximately $5-10 \mu \mathrm{m}$ and benefited from the wet etching process. The MPECVD procedure was initially heated and stabilized at $650^{\circ} \mathrm{C}$ and $10^{-5}$ torr; the CNTs grew slowly with increasing microwave power. A mixture gas of methane and hydrogen was then introduced into the deposition chamber through mass flow controllers. The microstructures of the electrodes and MWCNTs were examined by scanning electron microscopy, as shown in Figure 1a. Figure 1b is a cross-sectional image of another sample of the same batch. The morphology strongly depended on the growth conditions, especially temperature and input microwave power. It is obvious that the MWCNT grew directly between the two electrode pads with a nearly fixed length of $3 \mu \mathrm{m}$ and a diameter of $100 \mathrm{~nm}$ (even with some bends). Figure 1b clearly shows that the layered structure has $2.3 \mathrm{~nm} /$ per layer characteristics. The temperature dependence of resistivity in this individual MWCNT was then measured by the standard four-point probe method.

\section{Results and discussion}

According to the Mooij correlation in disordered metals, there will be a negative TCR when the resistivity of alloys or semiconductors is larger than $10-150 \mu \Omega-\mathrm{cm} .{ }^{23}$ This implies that a poorly conductive metal always behaves as a negative TCR material. That is why we presumed there would be a negative TCR phenomenon in this study.

The original TCR measurement data from the individual MWCNT with multiple defects formed on the body during the growth process is shown in Figure 2a. Resistivity would normally be linear with respect to temperature, ${ }^{24}$ but for quantities that vary polynomially or logarithmically with temperature, it may be possible to calculate a temperature coefficient that is a useful approximation for a certain range of temperatures. In order to visualize this behavior for TCR, Figure $2 \mathrm{~b}$ shows the logarithmic plot ver. $T^{-1}$ from Figure 2a. The dashed lines represent the fitting curves proportional to $T^{-1}$ under different slopes at both high temperatures (left side) and low temperatures (right side).

Clearly, there is a rapid, increasing trend with the decrease in temperature, especially below $50 \mathrm{~K}$. This represents a negative TCR $(\mathrm{d} \rho / \mathrm{dT}<0)$ phenomenon that cannot be explained by semiclassical theory. Figure $2 \mathrm{~b}$ seems to imply 

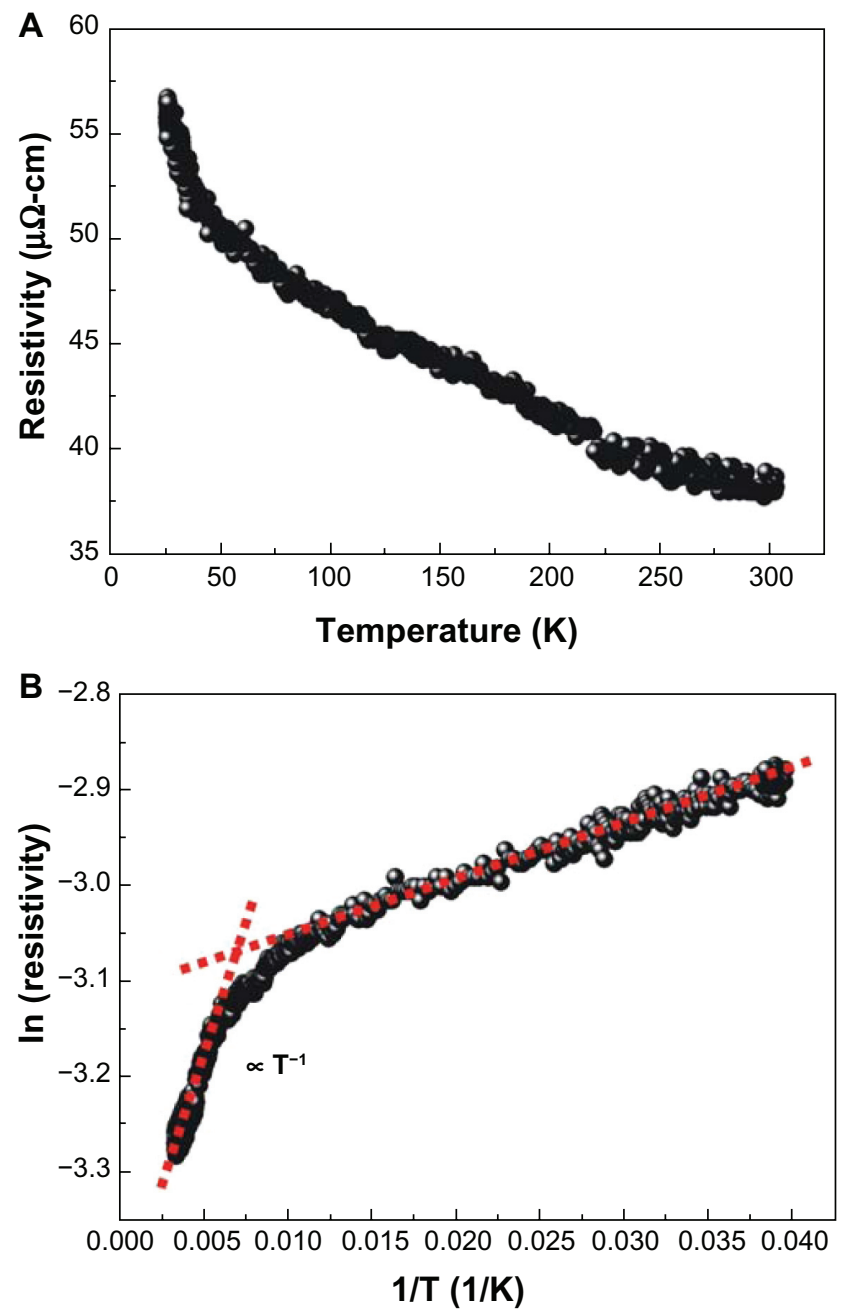

Figures 2 A) The results graph of the temperature dependence of resistivity of Figure I. B) Illustrated as a logarithmic graph, where dashed lines represent the fitting curve proportional to $T^{-1}$ under different slopes at high temperature (left side) and low temperature (right side).

that there would need to be two models to handle the variable trends of TCR with a transient point.

Other researchers have had similar results. Sheng reported that, for high-resistivity granular disordered systems, low-field conductivity $\exp \left(-\mathrm{A} / \mathrm{T}^{\alpha}\right)$ with $\alpha=1 / 2$ is obeyed over large temperature ranges, with possible crossovers to $\alpha=1 / 4$ at low temperatures and to $\alpha>1 / 2$ at high temperatures. ${ }^{25}$ The temperatures at which the crossovers occur depends on the distribution of grain size. As shown in Figure 3, Naeemi reported that, for single-wall and few-wall CNTs, the TCR reaches $1 /(\mathrm{T}-200 \mathrm{~K})$ for lengths much larger than the electron MFP. ${ }^{11}$ For MWCNTs with a large diameter $(>20 \mathrm{~nm})$, TCR varies from $-1 / \mathrm{T}$ to $+0.66 /$ (T-200K) as the length varies from zero to very large values. There exists a "singular point" around $200 \mathrm{~K}$ and a separate fitting model in two parts. The results were derived by simulations and assumed the body of the object would be

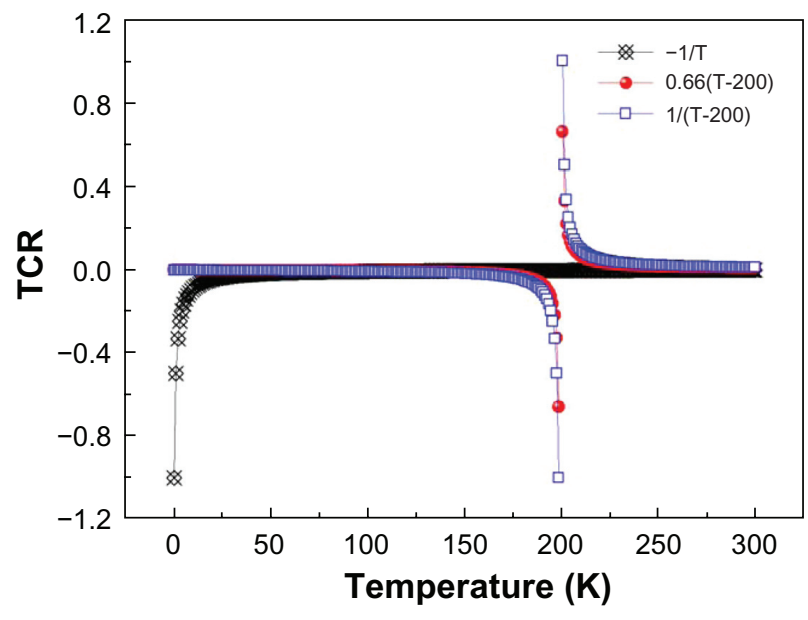

Figure 3 The work graph of Naeemi." Based on the physical and equivalent circuit model of carbon nanotubes, the temperature coefficient of resistance is always positive and increases with length. For single- and few-wall carbon nanotubes, it reaches I/(T-200K) for lengths much larger than the mean free path. For multiwalled carbon nanotubes with a large diameter $(>20 \mathrm{~nm})$, the temperature coefficient of resistance varies from $-\mathrm{I} / \mathrm{T}$ to $+0.66 /(\mathrm{T}-200 \mathrm{~K})$ as the length varies from zero to very large values.

perfect. However, what is different in a real MWCNT body which has multiple defects?

In our work, we employed hopping conductivity and variable range hopping conductivity theories to fit the experimental results for the two different slopes of Figure $2 b$.

In the hopping conductivity mechanism, the variation of resistivity at relatively high temperatures is in proportion to the Boltzman factor, $\exp (-\varepsilon / k T)$. Hopping conductivity theory, $\sigma=\sigma_{\min } \exp (-\varepsilon / k T)$, was used, as shown in Figure 4a, to display the fitting result curve. The resistivity $\sigma_{\min }$ derived from the fitting curve is $38 \mu \Omega-\mathrm{cm}$, and the corresponding activation energy $\varepsilon$ is $4.46 \mathrm{meV}$, which is quite close to the energy gap of SWCNT. ${ }^{26}$ Therefore, as the temperature increases, the thermal energy will excite an electron from the valance band to the conduction band, which leads to high conductivity.

However, the TCR at low temperature ranges cannot be explained by the exponential law above. Instead, when using strong localization theory $\left(\mathrm{k}_{\mathrm{f}} \mathrm{l}_{0} \sim 1\right)$, variable range hopping may prevail at low temperatures, as shown in Figure $4 \mathrm{~b}$. The exact variable range hopping theory that describes the lowtemperature behavior of resistivity in strongly disordered systems, where states are localized, can be expressed as:

$$
\boldsymbol{\sigma}=2 \boldsymbol{e}^{2} \boldsymbol{R}_{a v} \boldsymbol{N}\left(\boldsymbol{\varepsilon}_{\boldsymbol{F}}\right) \boldsymbol{v}_{p h} \exp \left(-\frac{\boldsymbol{B}}{\boldsymbol{T}^{1 / d+1}}\right)
$$

where $\boldsymbol{R}_{a v}$ is the average hopping distance, $\boldsymbol{N}\left(\boldsymbol{\varepsilon}_{F}\right)$ is the density of state, $v_{p h}$ is the coefficient according to the phonon spectrum, $\boldsymbol{B}$ is the coefficient related to the density of state, and $d$ indicates the dimension. 

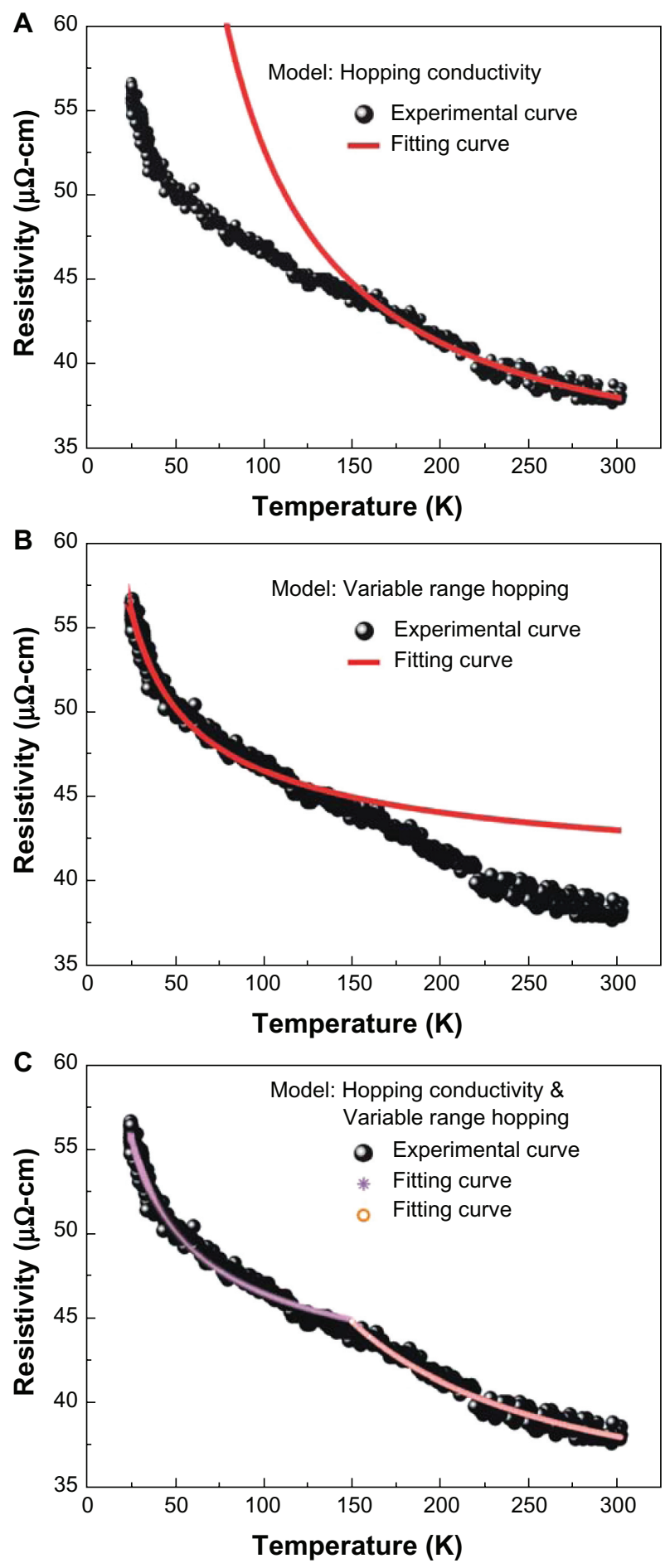

Figure 4 The fitting curve contrasted with real experimental data of a multiwalled carbon nanotube where curve (a) is obtained from hopping conductivity theory, curve (b) is obtained from variable range hopping theory, and curve (c) is the combined hopping conductivity and variable range hopping theories for the temperature dependence of resistivity.

Combining these two models, we can cover the temperature range from high to low $(300-25 \mathrm{~K})$, and note that there exists a singular point around $150 \mathrm{~K}$ (which is $50 \mathrm{~K}$ lower than in the work plot of Naeemi in Figure 3). ${ }^{11}$ This well-matched result is shown in Figure 4c, and explained
Table I The parameters obtained for fitting with hopping conductivity and variable range hopping models for a single multiwalled carbon nanotube

\begin{tabular}{llll}
\hline Model & Equation & $\mathbf{a}$ & $\mathbf{b}$ \\
\hline $\begin{array}{l}\text { Hopping } \\
\text { conductivity }\end{array}$ & $\sigma=a \times \exp \left(-\frac{b}{T}\right)$ & 0.031 & 51.74 \\
$\begin{array}{l}\text { Variable range } \\
\text { hopping }\end{array}$ & $\sigma=a \times \exp \left(-\frac{b}{\sqrt{T}}\right)$ & 0.038 & 1.90 \\
\hline
\end{tabular}

by taking into account the combined equations of hopping conductivity and variable range hopping conductivity theory. The fitting results are shown in Table 1, and are very consistent with Mott's predictions for the conductivity of amorphous materials. ${ }^{18}$

The meaning of the singular point here was presumed to be a transient point that governs the conditional change from inelastic to elastic collisions, ie, a measure of the temperature above which more modes of phonons in MWCNTs will be excited. To recognize and describe this unique property of MWCNTs, we named this singular point the "Lue point" temperature.

For comparison purposes, the grain boundary scattering model was also employed to fit for resistivity versus temperature in our individual MWCNT. The best fitting result is shown as a solid line in Figure 5, and makes the physical constraints of equation (6) with $p=1$ and $\boldsymbol{T}_{g}<1$.

In general, the value of $p$ depends on the dominant inelastic scattering mechanism, and is presumed to be fixed in the 0.5-4.0 temperature range..$^{27}$ The parameters obtained by fitting the R-T curves with the least square

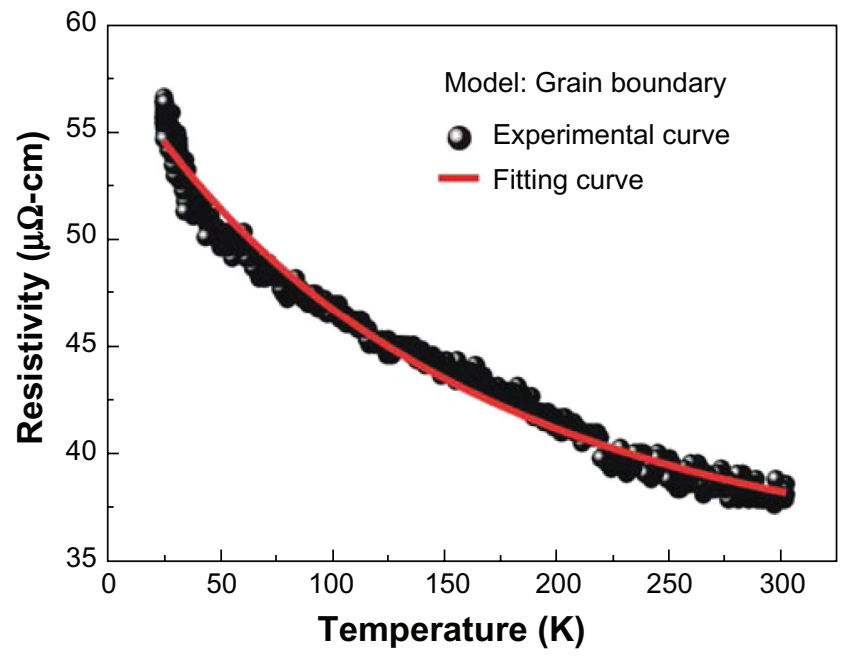

Figure $\mathbf{5}$ The fitting curve is obtained from grain boundary scattering theory and compared with real experimental data of an individual multiwalled carbon nanotube. 
Table 2 The parameters obtained for best fitting with grain boundary scattering model for a multiwalled carbon nanotube

\begin{tabular}{lllll}
\hline Model & $\frac{\boldsymbol{m} \boldsymbol{v}_{F}}{n_{\boldsymbol{n}} \boldsymbol{e}^{2} \boldsymbol{l}_{o}}\left(\frac{\boldsymbol{R}_{G}}{\boldsymbol{r}_{G}}\right)^{3}$ & $\mathbf{b}$ & $\frac{\boldsymbol{l}_{o}}{\boldsymbol{R}_{G}}$ & $\boldsymbol{T}_{\mathbf{g}}$ \\
\hline $\begin{array}{l}\text { Grain boundary } \\
\text { scattering }\end{array}$ & 5.614 & 0.0022 & 1.4 & 0.19 \\
\hline
\end{tabular}

errors method are listed in Table 2. The parameter term, $\boldsymbol{l}_{o} / \boldsymbol{R}_{G}$, indicates that the electrons are scattered by $\boldsymbol{l}_{o} / \boldsymbol{R}_{G}$ barriers in one mean free path $\left(\boldsymbol{l}_{\boldsymbol{o}}\right)$ where $\boldsymbol{R}_{G}$ is the interval between grains. According to the scanning electron microscopy image shown in Figure 1, the diameter of the MWCNT is smaller than approximately $100 \mathrm{~nm}$, with about $2.3 \mathrm{~nm} /$ per layer, therefore the elastic mean free path obtained by the fitting result of grain boundary scattering model is around 3-4 $\mathrm{nm}$.

Parameter $\boldsymbol{b}$ plays a crucial role and affects the tendency of the R-T curve, due to the mean free path's $\boldsymbol{l}(T)=\boldsymbol{l}_{\boldsymbol{o}} /\left(1+b \mathrm{~T}^{\mathrm{p}}\right)$ variance with temperature. According to Matthiessen's rule, $1 / \boldsymbol{l}(T)=1 / \boldsymbol{l}_{\boldsymbol{o}}+1 / \boldsymbol{l}_{\boldsymbol{i}}$, where $\boldsymbol{l}_{\boldsymbol{i} \text { }}$ is the mean free path due to the inelastic scattering which comes from the contribution of the optical phonon, we deduce that the interaction between the phonon and electron at low temperatures is small, ie, the inelastic mean free path varying with temperature causes the negative TCR characteristic in our study. In addition, the electron transmission probability, $\boldsymbol{T}_{g}$, for electrons to pass a single grain boundary is in the order of $10^{-1}$, as derived from the fitting result.

\section{Conclusion}

In this study, we have produced an individual MWCNT across electrodes by a method of MPECVD and measured the temperature dependence resistivity of this MWCNT to investigate its electronic properties.

For comparison, the effect of quantum confinement (strong localization) and grain boundary theories were quoted to explain the anomalous resistivity (a negative TCR characteristic). With regard to strong localization, the fitting result can be obtained by combining hopping conductivity theory in the high-temperature range and variable range hopping conductivity theory at the low-temperature range. The most appropriate fitting result is accomplished by using grain boundary theory. The singular "Lue point" temperature can be successfully described as a unique property of MWCNTs. The nanosize of the fabricated MWCNTs ensures the validity of the grain boundary scattering model and the temperature-related inelastic mean free path dominates the R-T curve, causing the negative TCR characteristic.

\section{Acknowledgments}

This work was supported by the National Science Council of the Republic of China under the contracts NSC-986-2112M007 and NSC 97-2221-E-029-007-.

\section{Disclosure}

The authors report no conflict of interest in this work.

\section{References}

1. Li J, Ye Q, Cassell A, et al. Bottom-up approach for carbon nanotube interconnects. Appl Phys Lett. 2003;82:2491-2493.

2. Saito R, Dresselhaus G, Dresselhaus MS. Physical Properties of Carbon Nanotubes. 5th ed. Singapore: Imperial College Press; 2003.

3. Anantram MP, Léonard F. Physics of carbon nanotube electronic devices. Rep Prog Phys. 2006;69:507-561.

4. Koentopp M, Chang C, Burke K, Car R. Density functional calculations of nanoscale conductance. J Phys Condens Matter. 2008;20:083203.

5. Ribaya BP, Leung J, Brown P, Rahman M, Nguyen CV. A study on the mechanical and electrical reliability of individual carbon nanotube field emission cathodes. Nanotechnology. 2008;19:185201.

6. Iijima S, Brabec C, Maiti A, Bernholc J. Structural flexibility of carbon nanotubes. J Chem Phys. 1996;104:2089-2092.

7. Chang LW, Lue JT. Electrical conductance in a single carbon wire. J Nanosci Nanotechnol. 2005;5:1-5.

8. Chen SY, Miao HY, Lue JT, Ouyang MS. Fabrication and field emission properties of multiwall nanotubes. J Phys D Appl Phys. 2004;37:273-279.

9. Liang W, Bockrath M, Bozovic D, Hafner JH, Tinkham M, Park H. Fabry-Perot interference in a nanotube electron waveguide. Nature. 2001;411:665-669.

10. Frank S, Poncharal P, Wang ZL, Heer WA. Carbon nanotube quantum resistors. Science. 1998;280:1744-1746.

11. Naeemi A, Meindl JD. Physical modeling of temperature coefficient of resistance for single- and multi-wall carbon nanotube interconnects. IEEE Electron Device Letters. 2007;28(2):135-138.

12. Ando T, Matsumura H, Nakanishi T. Theory of ballistic transport in carbon nanotubes. Physica B. 2002;323:44-50.

13. Miyamoto Y, Rubio A, Berber S, Yoon M, Tomanek D. Spectroscopic characterization of Stone-Wales defects in nanotubes. Phys Rev B. 2004;69:121413(R)

14. Pop E, Mann D, Reifenberg J, Goodson K, Dai H. Electro-thermal transport in metallic single-wall carbon nanotubes for interconnect applications. IEDM Technical Digest. 2005;253.

15. Svizhenko A, Anantram MP, Govindan TR. Ballistic transport and electrostatics in metallic carbon nanotubes. IEEE Transactions on Nanotechnology. 2005;4:557-562.

16. Chen SY, Chang LW, Peng CW, Miao HY, Lue JT. Growth of carbon nanotubes at low powers by impedance-matched microwave plasma enhanced chemical vapor deposition method. J Nanosci Nanotechnol. 2005;5:1887-1892.

17. Wang Y, Santiago-Avilés JJ. Large negative magnetoresistance and strong location in highly disordered electrospun pregraphitic carbon nanofiber. Appl Phys Lett. 2006;89:123119.

18. Mott NF, Davis EA. Electronic Processes in Non-Crystalline Materials. 2nd ed. Oxford: Clarendon Press; 1979.

19. C Godet. Variable range hopping revisited: The case of an exponential distribution of localized states. J Non-Cryst Solids. 2002;299-302: 333-338. 
20. Mayadas AF, Shatzkes M. Electrical-resistivity model for polycrystalline films: The case of arbitrary reflection at external surfaces. Phys Rev B 1970;1:1382-1389.

21. Vancea J, Hoffmann H, Kastner K. Mean free path and effective density of conduction electrons in polycrystalline metal films. Thin Solid Films. 1984;121:201.

22. Chen LJ, Tyan JH, Lue JT. The non-metallic conductivity of NbGe films sputtered on silicon substrates. J Phys Chem Solids. 1994;55:871-879.

23. Jayannavar AM, Kumar N. Mooij correlation in disordered metals. Phys Rev B. 1988;37(1):573-576.
24. Daou R, Nicolas DL, LeBoeuf D, et al. Linear temperature dependence of resistivity and change in the Fermi surface at the pseudogap critical point of a high-Tc superconductor. Nature Phys. 2009;5:31-34.

25. Sheng $P$, Klafter J. Hopping conductivity in granular disordered systems. Phys Rev B. 1983;27:2583-2586.

26. Ishibashi K, Moriyama S, Fuse T, Yamaguchi T. Carbon nanotubes as building blocks of quantum dots. Physica E. 2006;35:338-343.

27. Giordano N. Experimental study of localization in thin wires. Phys Rev B. 1980;22:5635-5655.

\section{Publish your work in this journal}

Nanotechnology, Science and Applications is an international, peerreviewed, open access journal that focuses on the science of nanotechnology in a wide range of industrial and academic applications. It is characterized by the rapid reporting across all sectors, including engineering, optics, bio-medicine, cosmetics, textiles, resource sustainability and science. Applied research into nano-materials, particles, nanostructures and fabrication, diagnostics and analytics, drug delivery and toxicology constitute the primary direction of the journal. The manuscript management system is completely online and includes a very quick and fair peer-review system, which is all easy to use.

Submit your manuscript here: http://www.dovepress.com/nanotechnology-science-and-applications-journal 